\title{
Theoretical Implications of the 47 Tuc Pulsars
}

\author{
Frederic A. Rasio \\ Department of Physics, MIT, Cambridge, MA 02139, USA
}

\begin{abstract}
Twenty millisecond radio pulsars have now been observed in the globular cluster 47 Tuc. This is by far the largest sample of radio pulsars known in any globular cluster. These recent observations provide a unique opportunity to re-examine theoretically the formation and evolution of recycled pulsars in globular clusters.
\end{abstract}

\section{Introduction}

The observations and properties of the 47 Tuc pulsars are presented by Camilo et al. (1999) and Freire et al., in these proceedings. All pulsars are clearly recycled, with pulse periods $P \simeq 2-8 \mathrm{~ms}$. At the time of the meeting, accurate timing solutions, including positions in the cluster, were known for 14 of the pulsars. The pulsars can be divided into 3 groups: 7 are single; 8 are in shortperiod binaries with orbital periods $P_{b}<0.5 \mathrm{~d} ; 5$ are in wider binaries with $P_{b}>1 \mathrm{~d}$ (see $\S 3$ ). The measured values of the period derivatives are probaly all determined predominantly by the pulsar accelerations in the cluster potential (9 out of 14 pulsars have $\dot{P}<0$ ). Under this assumption, Camilo et al. (1999) derive a central density $\rho_{c} \simeq 4 \times 10^{5} M_{\odot} \mathrm{pc}^{-3}$ using the method developed by Phinney (1993). This is somewhat larger than previous estimates (Pryor \& Meylan 1993 give $\rho_{c}=1.3 \times 10^{5} M_{\odot} \mathrm{pc}^{-3}$ ).

\section{Radial Distribution}

The radial distribution of the pulsars (Fig. 1) may appear surprising at first sight. Only 2 pulsars (47 Tuc O and L) are clearly inside the cluster core (assumed to have a radius $r_{c}=12^{\prime \prime}$, from the latest determination by De Marchi et al. 1996 based on HST WF/PC images). One pulsar (47 Tuc F) is near the edge of the core in projection, and all others are in the region $r / r_{c} \simeq 1-6$ (the outermost, 47 Tuc $\mathrm{C}$, has $r / r_{c}=5.6$ ). No pulsar is detected in the region $r / r_{c} \simeq 6-35$, even though this region is well covered by the Parkes beam (with a half-power diameter of about $14^{\prime}$ at $20 \mathrm{~cm}$ ).

The roughly flat histogram in $r$ (left panel of Fig. 1) suggests a deprojected $3 \mathrm{D}$ number density of pulsars $n(r) \propto r^{-2}$ out to $r \simeq 6 r_{c}$, while the absence of pulsars outside that region indicates a much steeper density profile for $r>6 r_{c}$. This is actually consistent with a thermally relaxed radial distribution in the cluster, not too different from that of other stellar components such as red giants (right panel of Fig. 1). This is to be expected since the central relaxation 

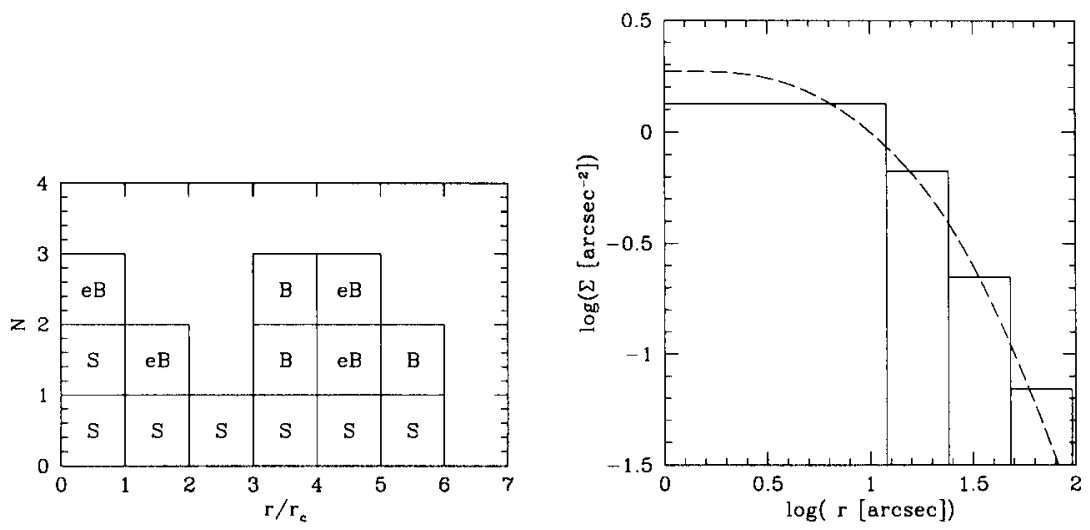

Figure 1. Projected radial distribution of the 47 Tuc pulsars. On the left is the histogram of the number of pulsars in each radial bin. The radius (distance to the cluster center) is given in units of the core radius $r_{c}=12^{\prime \prime}$ (from the HST WF/PC images of De Marchi et al. 1996). The labels have the following meaning: $S$ denotes a single pulsar, eB an "eclipsing binary" $\left(P_{b}<1 \mathrm{~d}\right)$, and B a "normal binary" $\left(P_{b}>1 \mathrm{~d}\right)$. On the right, the surface density of pulsars (histogram) is compared to that of giants (dashed line; from Fig. 2 of De Marchi et al. 1996). Pulsars were grouped into one core bin $\left(r / r_{c}=0-1\right)$ and 3 logarithmically spaced outer bins $\left(r / r_{c}=1-2,2-4\right.$, and $\left.4-8\right)$. The surface density of pulsars was rescaled to match that of giants at $\log \left(r\left[^{\prime \prime}\right]\right)=1.55$ (center of the third bin).

time in 47 Tuc, $t_{r c} \sim 10^{8} \mathrm{yr}$, is much shorter than typical characteristic ages of millisecond pulsars $\left(t_{c} \gtrsim 10^{9} \mathrm{yr}\right)$. A very similar type of radial distribution has been observed for the 7 single millisecond pulsars near the center of M15 (see Phinney 1993 for a detailed theoretical analysis). The small eccentricities of the binaries also indicate that the observed pulsars have not been perturbed by strong interactions with other cluster stars or binaries, consistent with their present positions in the cluster (see Rasio \& Heggie 1995). The concentration of wider binaries (47 Tuc $\mathrm{E}, \mathrm{H}$, and $\mathrm{Q}$ ) well outside the core, at $r \gtrless 3 r_{c}$, is also consistent with the smaller collision time in this region (the collision time for these binaries would have been $\sim 10^{9} \mathrm{yr}$ inside the core, but it increases to $\sim 10^{10} \mathrm{yr}$ at $r=3 r_{c}$ ). However, for the somewhat large eccentricity of $47 \mathrm{Tuc} \mathrm{H}$, $e=0.07$, to be explained by perturbations from other cluster stars, the results of Rasio \& Heggie (1995) show that this pulsar must have spent a significant fraction of its life inside the cluster core, in spite of its present location at $r=4 r_{c}$.

In a thermally relaxed stellar system, equipartition of energy leads to a more centrally concentrated spatial distribution for more massive stars. Since the main-sequence turnoff mass in the cluster ( $m_{\text {to }} \simeq 0.85 M_{\odot}$ for $47 \mathrm{Tuc}$ ) is a factor of $\sim 1.5-2$ lower than the masses of neutron star systems (including the companion masses for binaries), we would expect the radial distribution of pulsars to be more centrally concentrated than that of giants. This can be 


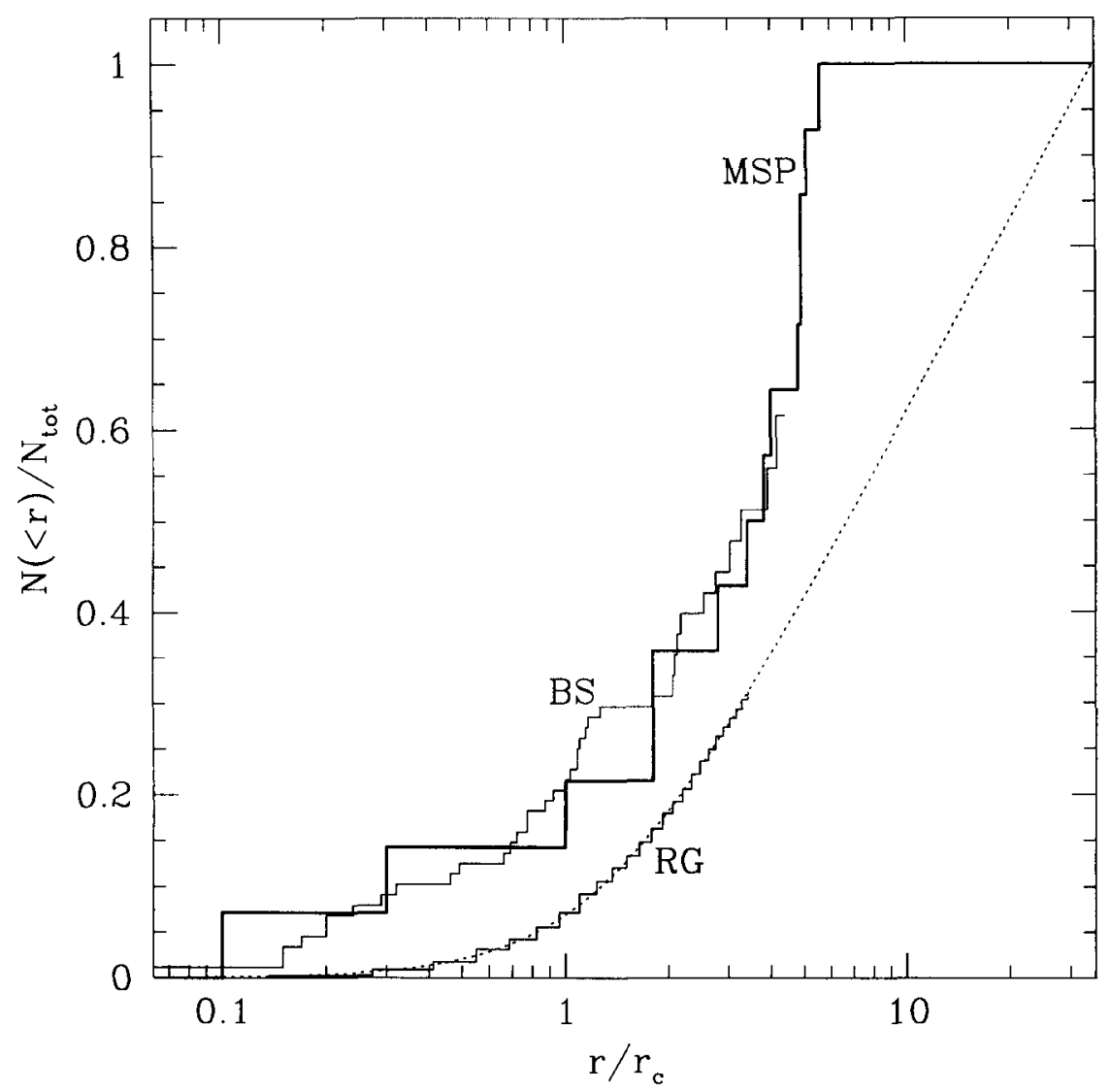

Figure 2. Cumulative radial distribution of the 47 Tuc pulsars (MSP, thick histogram), compared to that of red giants (RG) and blue stragglers (BS). The RG distribution was calculated by integrating the surface density profile given in Table 1 of De Marchi et al. (1996). The dotted line is the best fit to a modified Hubble law (which is analytic and closely approximates the inner regions of high-concentration King models; see, e.g., Binney \& Tremaine 1987), extended to $r=35 r_{c}$ (near the edge the Parkes beam at $20 \mathrm{~cm}$ ). Note that fitting the cumulative distribution leads to a tighter constraint on $r_{c}$ than fitting the surface density (as done by De Marchi et al.). The best-fit model shown here gives a core radius about $30 \%$ larger than that quoted by De Marchi et al. (although we still give $r$ in units of $r_{c}=12^{\prime \prime}$, as in Fig. 1). The BS distribution was obtained by combining the data sets of Paresce et al. (1991, HST FOC) and Guhathakurta et al. (1992, HST PC). Star HST-12 of Paresce et al. was adopted as the cluster center. The surface density at $r \gtrsim 2 r_{c}$ was corrected for incomplete radial annuli in the HST PC irnage (as in De Marchi et al. 1996). The BS distribution was normalized to match that of the millisecond pulsars at $r \simeq 4.2 r_{c}$ (extent of the HST PC image). 
seen most convincingly by comparing integrated surface density profiles (Fig. 2). HST observations of the central region of 47 Tuc have revealed a large number of centrally concentrated, bright blue stragglers (Paresce et al. 1991; Guhathakurta et al. 1992), which are thought to be formed through collisions and mergers of main-sequence stars in the dense cluster core (Lombardi et al. 1996; Sills et al. 1999). These blue stragglers are main-sequence stars well above the turnoff, with theoretically derived masses in the range $m_{b s} \simeq 1.4-1.7 M_{\odot}$ (one has a directly measured mass of $1.7 \pm 0.4 M_{\odot}$; see Shara et al. 1997), i.e., very similar to the masses of neutron star systems. Indeed, we see in Fig. 2 that the radial distributions of blue stragglers and millisecond pulsars in 47 Tuc appear strikingly similar.

\section{Formation of Recycled Pulsars in Short-Period Binaries}

The binary properties of the 47 Tuc pulsars are quite surprising. While 7 pulsars are single, the majority are in short-period binaries. Most of the binaries ( 8 out of 13) have properties similar to those of the rare "eclipsing binary pulsars" (5 do indeed show evidence for radio eclipses) seen in the Galactic disk population (see Nice, these proceedings, for a review). These systems have extremely short orbital periods, $P_{b} \sim 1-10 \mathrm{hr}$, circular orbits, and very low-mass companions, with $m_{2} \sin i \sim 0.01-0.1 M_{\odot}$. The remaining 5 binaries have properties more similar to those of the bulk disk population, with nearly-circular orbits, periods $P_{b} \sim 1-3 \mathrm{~d}$ (near the short-period end of the distribution for binary millisecond pulsars in the disk) and companions of mass $m_{2} \sin i \simeq 0.2 M_{\odot}$ (presumed to be white dwarfs, hereafter WD). The large inferred total population of recycled pulsars in 47 Tuc ( $\sim 10^{3}$, see Camilo et al. 1999), as well as the high central density of the cluster, suggest that dynamical interactions must play a dominant role in the formation of these systems. However, the two dynamical formation scenarios traditionally invoked for the production of recycled pulsars in globular clusters run into many difficulties.

Scenarios based on tidal capture of low-mass main-sequence stars (MS) by neutron stars (NS), followed by accretion and recycling of the NS during a stable mass-transfer phase, fell out of favor many years ago. Serious problems were pointed out about the tidal capture process itself (which, because of strong nonlinearities in the regime relevant to globular clusters, is far more likely to result in a merger than in the formation of a detached binary; see, e.g., Kumar \& Goodman 1996; McMillan et al. 1990; Rasio \& Shapiro 1991; Ray et al. 1987). Moreover, the basic predictions of tidal capture scenarios are at odds with many observations of binaries in clusters (Bailyn 1995; Johnston et al. 1992; Shara et al. 1996). It is likely that "tidal-capture binaries" are either never formed, or contribute negligibly to the production of recycled pulsars. Verbunt (1987) proposed that collisions between NS and red giants might produce directly NSWD binaries with ultra-short periods, but detailed hydrodynamic simulations later showed that this does not occur (Rasio \& Shapiro 1991).

The viability of tidal capture and two-body collision scenarios has become less relevant with the realization over the last 10 years that globular clusters contain dynamically significant populations of primordial binaries (Hut et al. 1992). Neutron stars can then acquire MS companions through exchange interactions 
with these primordial binaries. Because of its large cross section, this process dominates over any kind of two-body interaction even for low primordial binary fractions (Heggie et al. 1996; Leonard 1989; Sigurdsson \& Phinney 1995). In contrast to tidal capture, exchange reactions with hard primordial binaries (with semimajor axes $a \sim 0.1-1 \mathrm{AU}$ ) can form naturally the wide binary millisecond pulsars seen in some low-density globular clusters (such as PSR B1310+18, with $P_{b}=256 \mathrm{~d}$, in M53, which has the lowest central density, $\rho_{c} \sim 10^{3} M_{\odot} \mathrm{pc}^{-3}$, of any globular cluster with observed radio pulsars; see, e.g., Phinney 1996). When the newly acquired MS companion, of mass $\lesssim 1 M_{\odot}$, evolves up the giant branch, the orbit circularizes and a period of stable mass transfer begins, during which the NS is recycled (see, e.g., Rappaport et al. 1995). The resulting NS-WD binaries have orbital periods in the range $P_{b} \sim 1-10^{3} \mathrm{~d}$. However, this scenario cannot explain the formation of recycled pulsars in binaries with periods shorter than $\sim 1 \mathrm{~d}$. To obtain such short periods, the initial primordial binary must be extremely hard, with $a \lesssim 0.01 \mathrm{AU}$, but the recoil velocity of the system following the exchange interaction would then almost certainly exceed the escape speed from the shallow cluster potential $\left(v_{e} \simeq 60 \mathrm{~km} \mathrm{~s}^{-1}\right.$ for $47 \mathrm{Tuc}$ ).

One can get around this problem by considering more carefully the stability of mass transfer in NS-MS binaries formed through exchange interactions. While all MS stars in the cluster today have masses $\lesssim 1 M_{\odot}$, the rate of exchange interactions may very well have peaked at a time when significantly more massive MS stars were still present. Indeed, the NS and the most massive primordial binaries will undergo mass segregation and concentrate in the cluster core on a time scale comparable to the initial half-mass relaxation time $t_{r h}$. For a dense cluster like 47 Tuc, we expect $t_{r h} \simeq 10^{9} \mathrm{yr}$ (slightly lower than the present value), which is comparable to the MS lifetime of $\mathrm{a} \simeq 2-3 M_{\odot}$ star. If the majority of NS acquired these more massive companions, a drastically different evolution follows. Indeed, in this case, when the MS star evolves and fills its Roche lobe, the mass transfer is dynamically unstable and leads to a common-envelope (CE) phase. The emerging binary will have a low-mass WD in a short-period, circular orbit around the NS. This simple idea is at the basis of the dynamical scenario developed recently by Rasio, Pfahl, \& Rappaport (1999). They find that a significant fraction of the NS-WD binaries emerging from the CE phase undergo further evolution during a period of stable mass transfer driven by gravitational radiation and tidal heating of the companion. The properties of the final binaries match very well the observed properties of very short-period (eclipsing) binaries in 47 Tuc. A similar scenario, but starting from tidal capture binaries and applied to X-ray sources in globular clusters, was discussed by Bailyn \& Grindlay (1987). The possibility of forming intermediate-mass binaries through exchange interactions was mentioned by Davies \& Hansen (1998), who pointed out that NS retention in globular clusters may also require that the NS be born in massive binaries. Among eclipsing pulsars in the disk, at least one system (PSR J2050-0827) is likely to have had an intermediate-mass binary progenitor, given its very low transverse velocity (Stappers et al. 1998).

Acknowledgments. This work was supported by NSF Grant AST-9618116 and NASA ATP Grant NAG5-8460, and by a Sloan Research Fellowship. 


\section{References}

Bailyn, C.D. 1995, ARAA, 33, 133

Bailyn, C.D., \& Grindlay, J.E. 1987, ApJ, 316, L25

Binney, J., \& Tremaine, S. 1987, Galactic Dynamics (Princeton Univ. Press)

Camilo, F., Lorimer, D.R., Freire, P., Lyne, A.G., \& Manchester, R.N. 1999, ApJ, submitted

Davies, M.B., \& Hansen, B.M.S. 1998, MNRAS, 301, 15

De Marchi, G., Paresce, F., Stratta, M.G., Gilliland, R.L., \& Bohlin, R.C. 1996, ApJ, L51

Guhathakurta, P., Yanny, B., Schneider, D.P., \& Bahcall, J.N. 1992, AJ, 104, 1790

Heggie, D.C., Hut, P., \& McMillan, S.L.W. 1996, ApJ, 467, 359

Hut, P., et al. 1992, PASP, 104, 981

Johnston, H.M., Kulkarni, S.R., \& Phinney, E.S. 1992, in X-Ray Binaries and Recycled Pulsars, eds. E.P.J. van den Heuvel \& S.A. Rappaport (Dordrecht: Kluwer), 349

Kumar, P., \& Goodman, J. 1996, ApJ, 466, 946

Leonard, P.J.T. 1989, AJ, 98, 217

Lombardi, J.C., Rasio, F.A., \& Shapiro, S.L. 1996, ApJ, 468, 797

McMillan, S.L.W., Taam, R.E., \& McDermott, P.N. 1990, ApJ, 354, 190

Paresce, F., et al. 1991, Nature, 352, 297

Phinney, E.S. 1993, in Structure and Dynamics of Globular Clusters, eds. S.G. Djorgovski \& G. Meylan (ASP Conf. Ser. Vol. 50), 141

Phinney, E.S. 1996, in The Origins, Evolutions, and Destinies of Binary Stars in Clusters, eds. E.F. Milone \& J.-C. Mermilliod (ASP Conf. Ser. Vol. 90), 163

Pryor, C., \& Meylan, G. 1993, in Structure and Dynamics of Globular Clusters, eds. S.G. Djorgovski \& G. Meylan (ASP Conf. Ser. Vol. 50), 357

Rappaport, S., Podsiadlowski, P., Joss, P.C., Di Stefano, R., \& Han, Z. 1995, MNRAS, 273, 731

Rasio, F.A., \& Heggie, D.C. 1995, ApJ, 445, L133

Rasio, F.A., Pfahl, E.D., \& Rappaport, S. 1999, ApJ, submitted

Rasio, F.A., \& Shapiro, S.L. 1991, ApJ, 377, 559

Ray, A., Kembhavi, A.K., Antia, H.M. 1987, A\&A, 184, 164

Shara, M.M., Bergeron, L.E., Gilliland, R.L., Saha, A., \& Petro, L. 1996, ApJ, 471,804

Shara, M.M., Saffer, R.A., \& Livio, M. 1997, ApJ, 489, L59

Sigurdsson, S., \& Phinney, E.S. 1995, ApJS, 99, 609

Sills, A., Bailyn, C.D., Edmonds, P., \& Gilliland, R. 1999, ApJ, submitted

Stappers, B.W., Bailes, M., Manchester, R.N., Sandhu, J.S., \& Toscano, M. 1998, ApJ, 499, L183

Verbunt, F. 1987, ApJ, 312, L23 